\title{
Identificación de las fortalezas y debilidades de la competencia digital en el uso de aplicaciones de internet del alumno de primer curso del Grado de Magisterio
}

\section{Identifying the strengths and weaknesses of the digital competency in the use of Internet applications in first grade of the Teacher Degree}

\author{
Marta LIESA ORÚS, Sandra VÁZQUEZ TOLEDO y Jorge LLORET GAZO
}

Universidad de Zaragoza

Recibido: Febrero 2015

Evaluado: Marzo 2015

Aceptado: Abril 2015

\begin{abstract}
Resumen
El objetivo de este estudio se centra en identificar la competencia digital en el uso de aplicaciones de internet en los estudiantes del primer curso del Grado de Maestro en Educación Infantil y Primaria de la Universidad de Zaragoza del curso académico 2014-2015, un total de 960 alumnos, de los cuales 240 estaban matriculados en la Facultad de Ciencias Humanas y de la Educación de Huesca, 240 en la Facultad de Ciencias Sociales y Humanas de Teruel y 480 en la Facultad de Educación de Zaragoza. Para ello se aplicó un cuestionario de elaboración propia, realizado por profesores del Departamento de Ciencias de la Educación, del Departamento de Informática e Ingeniería de Sistemas de la Universidad de Zaragoza y por profesores del área de Tecnología y del área de Matemáticas de dos Institutos de Educación Secundaria de la Comunidad Autónoma de Aragón. Los resultados obtenidos indican que los estudiantes que llegan a la Universidad, a pesar de ser de la generación de la era digital, tienen debilidades importantes en el uso de las herramientas de internet que analizamos en este estudio. El análisis cualitativo de los resultados muestra que la mayoría de los estudiantes conocen y usan frecuentemente tan solo cuatro de las veinte herramientas sobre las que se les pregunta en esta investigación.Palabras clave: alfabetización digital, educación superior, competencias del maestro, herramientas de internet.
\end{abstract}

\footnotetext{
Abstract

The aim of this research is to identify the digital competence in the use of internet tools in firstyear students of Preschool and Primary Education Teacher Training Degree at the University of Zaragoza in the academic year 2014-2015. The sample included a total of 960 students, from which 240 were enrolled in the Faculty of Human Sciences and Education of Huesca, 240 in the Faculty of Social and Human Sciences of Teruel and 480 in the Faculty of Education of Zaragoza). They were given a questionnaire, specifically designed by teachers of the Departments of Educational Sciences, Computer Science and Systems Engineering of the 
University of Zaragoza and by teachers of Technology and of Mathematics of two Secondary Education Schools of the Autonomous Community of Aragon. The results indicate that students arriving at University for the first time, despite belonging to the digital era, have serious weakness in digital skills, in the use of the internet tools analyzed in this study. The qualitative analysis of the results shows that the majority of students knows and uses frequently only four of twenty tools considered in this research.

Keywords: computer literacy, higher education, teacher competencies, internet tools.

La sociedad se ha transformado, ha sido sometida a cambios muy importantes en el ámbito social, económico y tecnológico, a los que la escuela tiene que dar respuesta. Uno de los aspectos en los que la escuela debe avanzar es en el desarrollo de la competencia digital en sus alumnos y alumnas, y en el uso de aplicaciones de Internet con carácter didáctico a través de la introducción en sus aulas de las nuevas tecnologías como formas de comunicación y acceso a la información. Los usuarios de las escuelas, niños y jóvenes, también han cambiado sus formas de relacionarse y de buscar información a través de internet y las han integrado en su vida cotidiana.

La competencia digital que tiene como principio introducir e integrar las TIC en los procesos de enseñanza aprendizaje, ha sido considerada en Europa como una competencia esencial a trabajar desde la escuela, y así lo ha asumido nuestro sistema educativo a través de las legislaciones nacionales y autonómicas.

Las competencias básicas que llegan a nuestro sistema educativo con la LOE (2006) son uno de los elementos que conforman el currículum. En el preámbulo de dicha ley, se hace referencia a las propuestas que hace la Unión Europea y la UNESCO en relación con la necesidad de potenciar la competencia digital para mejorar la calidad y la eficacia de los sistemas educativos. La prescripción normativa señala que las TIC se trabajarán en todas las áreas, permitiendo desarrollar su carácter transversal (art. 19), tal y como recogen los principios pedagógicos de la LOE.

En el Real Decreto 1513/2006, de 7 de diciembre, en el que se establecen las enseñanzas mínimas de la Educación Primaria, se desarrollan las ocho competencias que el alumno(a) tiene que adquirir durante su educación en esta etapa, incluida la competencia digital. Esta competencia queda recogida tanto en la Educación Primaria como Secundaria, sin embargo, a pesar de su importancia y significación, en ningún texto legal se contempla explícitamente dentro de la Educación Infantil (Muñoz, 2010). En el Real Decreto 1630/2006, de 29 de diciembre por el que se establece el currículo y las enseñanzas mínimas del segundo ciclo de la Educación Infantil no la contempla explícitamente, aunque sí que en el área de Comunicación y Representación y en el área de Conocimiento del Entorno se hace referencia a la importancia de las TIC en esta etapa educativa y a la necesidad de empezar a introducir su uso en la escuela. Así mismo, el informe de 2011 realizado por el Departamento de Proyectos Europeos del Instituto de Tecnologías Educativas sobre competencia digital, pone de manifiesto la importancia de incorporar las TIC en el aula tan pronto como sea posible, ya desde el nivel de Educación Infantil, de una manera global y con el objetivo de que los estudiantes puedan utilizar las herramientas digitales de una manera crítica. (Marín Díaz, 2012) 
En el currículum de Aragón se recoge dicha competencia en la Orden de 28 de marzo de 2008, por la que se aprueba el currículo de la Educación infantil, y en la Orden de 16 de junio de 2014 (art.6), por la que se aprueba el currículum de la Educación Primaria y se autoriza su aplicación en los centros de la Comunidad Autónoma de Aragón.

La LOMCE sigue hablando igualmente de competencia digital, se introduce en el currículum, como competencia clave y como elemento transversal, las competencias relacionadas con las TIC deben trabajarse desde todas las asignaturas del currículum. En el artículo 111 (Tecnologías de la Información y la Comunicación) detalla algunas propuestas para trabajar las TIC desde las escuelas, al hablar de: interoperabilidad, entornos virtuales, contenidos digitales, plataformas de recursos digitales de aprendizaje, del marco común de referencia de competencia digital docente para orientar la formación del profesorado y de los centros, así como de un uso de las TIC en el aula como medio didáctico apropiado para tareas de enseñanza y aprendizaje. Y en el punto 3 del artículo 122 (Acciones destinadas a fomentar la calidad de los centros docentes) establece que un proyecto educativo de calidad supondrá la especialización de los centros docentes, que podrá comprender, entre otras actuaciones la aportación de recursos didácticos a plataformas digitales compartidas.

En cualquier caso independientemente de los cambios legislativos que sigamos sufriendo en educación, no cabe duda del papel que las TIC desempeñan en nuestra sociedad y por tanto de la necesidad de ser tratadas desde el ámbito educativo.

\section{La formación del maestro en competencia digital}

La capacitación del profesorado y el acceso a las TIC se consideran condiciones imprescindibles para mejorar la calidad y eficacia de la educación (Gutiérrez, 2008). Las TIC pueden ayudar al profesorado a seguir, regular y controlar los progresos y las dificultades de los estudiantes, así como para apoyar el proceso de retroalimentación y orientar su aprendizaje (Coll, Mauri y Onrubia, 2008). En el preámbulo de la LOE y en el de la LOCME se apunta a la necesidad de mejorar la calidad y la eficacia de los sistemas educativos, mejorando la formación del profesorado a través del desarrollo de las aptitudes necesarias para la sociedad del conocimiento y de garantizar el acceso de todos a las tecnologías de la información y la comunicación.

Hay muchos estudios sobre las competencias y contenidos que deben trabajarse en la formación del profesorado relacionadas con las TIC, Bautista (2000), Marqués (2003), Cabero (2004), Area (2005), entre otros. La mayoría de los autores entienden que formar a los profesores(as) únicamente en la dimensión técnica es insuficiente y que hay que añadir formación en la dimensión didáctica y educativa (Gutiérrez, 2008). Si no hacemos esto, estaremos cayendo en las Facultades en lo que algunos autores denominan "el enfoque instrumentalista centrado en el uso correcto del artefacto" (Granados, 2007, 135) y olvidando otras dimensiones pedagógicas mucho más importantes. 
La dimensión didáctica en el uso de las TIC es la realmente importante en la formación del maestro(a), y forma parte de las competencias que todo docente tiene que tener adquiridas al terminar su carrera, tal y como recoge la Memoria de Verificación del Grado de Magisterio en Educación Infantil y la de Educación Primaria de la Universidad de Zaragoza. Estas competencias se trabajan desde distintas materias y asignaturas, en el Grado de Educación Infantil, concretamente se trabajan en la asignatura "Materiales y Recursos" en segundo curso, y en el Grado de Educación Primaria se trabajan en la asignatura "La educación en la Sociedad del Conocimiento" que se imparte en el primer curso de la carrera.

En concreto, en las asignaturas mencionadas se trabajan contenidos relacionados con la formación de las TIC en el aula y en el hogar, con la importancia de las TIC en la sociedad de la información, contenidos relacionados con la Historia de las TIC, la "brecha digital, la "alfabetización digital”, el "imperativo tecnológico". Se estudian también productos educativos multimedia para el aula: presentaciones, páginas Web, ebook, tutoriales interativos, vídeo digital, podcast, enciclopedias electrónicas, kits de construcción, simuladores, blogs, wikis, redes sociales y entornos colaborativos, conocimiento compartido, videoconferencia, pasatiempos educativos, webquest, foros, buscadores, etc. Así mismo, se trabajan contenidos conceptuales como "interactividad" y "navegación". Se analizan los valores y adicciones en la televisión, juegos, videoconsolas y redes sociales. Y se estudian también algunos aspectos de la tecnoética. Además se enseñan programas ofimáticos, se enseña a usar la pizarra digital interactiva, el TabletPC-netbook-PDA, el proyector, la cámara digital de fotos, la cámara de videoconferencia, la cámara de documentos. Se les enseña que existen distintos tipos de ratones y teclados,.... redes alámbricas e inalámbricas, software y partes esenciales del ordenador: antivirus, cortafuegos, control parental, copias de seguridad,..., discos, periféricos y almacenamiento, entre otros contenidos.

Como puede verse la formación actual del titulado en Magisterio va orientada tanto a los aspectos tecnológicos como a los aspectos metodológicos y sociales de la integración de estos recursos en su práctica docente cotidiana. Entendemos que la capacitación tecnológica ha de verse siempre como parte de la formación didáctica, o integrada en el desarrollo de un proyecto educativo (Gutiérrez, 2003).

En este sentido, la formación del profesorado debe incluir la competencia digital enfocada a la enseñanza, y no basada únicamente en formar para el uso de las TIC (Ala-Mutka; Punie y Redecker, 2008; Barrantes, Casas y Luengo, 2011). Es necesario que el maestro(a) sea consciente de la importancia que tiene esta competencia en la sociedad actual y trabaje de forma innovadora, para incorporar las TIC a la vida del aula e integrarlas en las actividades diarias de sus alumnos(as), aprovechando todas las posibilidades que éstas brindan a la enseñanza y al aprendizaje.

Con esta investigación que se presenta en este artículo se pretende saber qué conocimientos previos tienen los estudiantes de Magisterio tanto del Grado de Educación Infantil como los del Grado de Educación Primaria de las aplicaciones de Internet, para posteriormente darles las pinceladas pedagógicas a través de la asignatura de Materiales y Recursos en el Grado de Educación Infantil y La Escuela en la Sociedad del Conocimiento en el Grado de Educación Primaria. 
La generación de los estudiantes que han formado parte de este estudio, serán de la promoción de Maestros egresados del curso 2018-2019, y nacieron en su gran mayoría en el año 1996-1997. Han sido ya nativos digitales, pues el nacimiento de Internet como una red de comunicación disponible en la mayoría de las sociedades desarrolladas data de finales de los años noventa, y coincide con el nacimiento de estos jóvenes que hoy día están accediendo a la Universidad. Además, siguiendo la terminología de Prensky (2001), también son inmigrantes digitales, pues el maestro(a) recibe formación sobre las TIC ya sea en la titulación del Grado o en su formación continua posterior, cuando ya cuenta con una capacitación en el dominio y análisis de las herramientas que le permite acceder al mundo digital con cierto espíritu crítico.

Somos conscientes de la importancia de que los profesores(as) desarrollen la competencia digital con sentido crítico y pedagógico. Por eso como ya se ha dicho anteriormente, el objetivo de este estudio se centra en identificar la competencia digital en el uso de aplicaciones de Internet de los estudiantes de primer curso del Grado de maestro en Educación Infantil y Primaria de la Universidad de Zaragoza (un total de 960 alumnos). Se pretende conocer y analizar las fortalezas y debilidades que tienen en el uso de aplicaciones de Internet que sería importante que dominaran cuando terminaran el Grado, para conocer con qué conocimientos previos llegan del Bachillerato o de los estudios previos con los que accedan a la titulación y poder complementar dichos conocimientos en las asignaturas de la carrera. Nos interesa saber qué fortalezas y debilidades tienen en el uso de los navegadores, los compresores de archivos, el uso del correo electrónico, la mensajería instantánea, el uso de los foros, los blogs, las wikis, la nube, llamadas telefónicas por internet, redes sociales profesionales, el microblogging, etc. Queremos saber si conocen lo que es cada uno de estos elementos, si los usan y si su uso es esporádico o frecuente, pues estamos convencidos que en su futuro profesional como docentes el uso de todas estas herramientas puede serles de gran utilidad.

\section{Aplicaciones internet en la sociedad actual}

En lo que se refiere a las herramientas que se han evaluado en este estudio realizado en la Universidad de Zaragoza, éstas se recogen en la Tabla 1 junto a una breve descripción de las mismas. Se han seleccionado estas herramientas y no otras, porque son actualmente las aplicaciones de uso de Internet más importantes, según el criterio de los autores de este estudio.

\begin{tabular}{ll}
\hline Herramienta & \multicolumn{1}{c}{ Descripción } \\
\hline Navegador & $\begin{array}{l}\text { Acceso a los sitios web. Algunos ejemplos de navegadores son Internet } \\
\text { Explorer, Mozilla Firefox o Google Chrome }\end{array}$ \\
\hline $\begin{array}{l}\text { Compresor de } \\
\text { archivos }\end{array}$ & $\begin{array}{l}\text { Comprime uno o varios archivos en uno solo de forma que su tamaño es menor } \\
\text { que la suma de los tamaños de los archivos originales. Algunos compresores son } \\
\text { WinZip WinRar o 7-zip }\end{array}$ \\
\hline $\begin{array}{l}\text { Correo } \\
\text { electrónico }\end{array}$ & $\begin{array}{l}\text { Envía mensajes a través de la red. Algunos de los proveedores de correo más } \\
\text { conocidos son Otulook, Gmail o Yahoo }\end{array}$ \\
\hline $\begin{array}{l}\text { Mensajería } \\
\text { instantánea }\end{array}$ & Permite establecer conversaciones escritas. Un ejemplo de programa es Skype \\
\hline Foros & Sitio donde se buscan respuestas a preguntas o se plantean nuevas preguntas \\
\hline
\end{tabular}




\begin{tabular}{|c|c|}
\hline $\begin{array}{l}\text { Grupos de } \\
\text { noticias }\end{array}$ & $\begin{array}{l}\text { Nos envía noticias, mediante correo electrónico, sobre un determinado tema al } \\
\text { que nos hemos suscrito previamente }\end{array}$ \\
\hline $\begin{array}{l}\text { Listas de } \\
\text { distribución }\end{array}$ & Análogo a los grupos de noticias \\
\hline $\begin{array}{l}\text { Editores de } \\
\text { HTML }\end{array}$ & $\begin{array}{l}\text { Crea sitios web usando HTML, el lenguaje con el que se construyen los sitios } \\
\text { web. Un ejemplo de esta herramienta es Adobe Dreamweaver CC }\end{array}$ \\
\hline Blogs & $\begin{array}{l}\text { Publica entradas ordenadas temporalmente de manera secuencial. Ejemplos de } \\
\text { sitios para construir blogs son Blogger o Wordpress }\end{array}$ \\
\hline Fuentes web & $\begin{array}{l}\text { Recopila las entradas publicadas en diversas fuentes de información, como } \\
\text { periódicos o blogs. Un ejemplo de programa de fuentes web es Feedly }\end{array}$ \\
\hline Wikis & $\begin{array}{l}\text { Permite el trabajo colaborativo de los usuarios ya que pueden compartir } \\
\text { información en forma de páginas web y de ficheros }\end{array}$ \\
\hline $\begin{array}{l}\text { Editor de texto en } \\
\text { la nube }\end{array}$ & \multirow[t]{3}{*}{$\begin{array}{l}\text { Realiza tareas ofimáticas y guarda los ficheros en la nube. Algunos ejemplos son } \\
\text { Google Docs u Office Online }\end{array}$} \\
\hline $\begin{array}{l}\text { Hoja de cálculo } \\
\text { en la nube }\end{array}$ & \\
\hline $\begin{array}{l}\text { Presentación en la } \\
\text { nube }\end{array}$ & \\
\hline $\begin{array}{l}\text { Llamadas } \\
\text { telefónicas } \\
\text { por Internet }\end{array}$ & Permite hacer llamadas gratuitas. Un programa es Skype \\
\hline $\begin{array}{l}\text { Redes sociales } \\
\text { generalistas }\end{array}$ & Nos ponen en contacto con amigos. Un ejemplo es el sitio Facebook \\
\hline $\begin{array}{l}\text { Redes sociales } \\
\text { profesionales }\end{array}$ & $\begin{array}{l}\text { Nos ponen en contacto con empresas que ofrecen puestos de trabajo. Un ejemplo } \\
\text { es el sitio LinkedIn }\end{array}$ \\
\hline Microblogging & $\begin{array}{l}\text { Publica entradas secuenciales con un límite de longitud. Por ejemplo, Twitter } \\
\text { donde el límite de cada entrada son } 140 \text { caracteres. }\end{array}$ \\
\hline $\begin{array}{l}\text { Comunidades de } \\
\text { contenidos }\end{array}$ & $\begin{array}{l}\text { Recopilan contenidos de diversos tipos. Por ejemplo, fotos en Flickr o en } \\
\text { Instagram }\end{array}$ \\
\hline Etiquetado social & $\begin{array}{l}\text { Comparte contenidos y se etiqueta para dar a conocer de qué tratan esos } \\
\text { contenidos. Un ejemplo es el sitio web delicious.com }\end{array}$ \\
\hline
\end{tabular}

Tabla 1. Herramientas evaluadas y breve descripción de las mismas

\section{Método}

\section{Participantes}

En el estudio participaron todos los estudiantes de primer curso del Grado en Magisterio de Educación Infantil y Primaria de la Universidad de Zaragoza de sus tres campus, 240 de la Facultad de Ciencias Humanas y de la Educación de Huesca, 240 de la Facultad de Ciencias Sociales y Humanas de Teruel y 480 de la Facultad de Educación de Zaragoza, un total de 960 estudiantes. De esta muestra el 80\% de los alumnos(as) tenían entre los 18 y los 23 años.

\section{Instrumento de evaluación}

Para la recogida de los datos se utilizó un cuestionario elaborado por profesores del Departamento de Ciencias de la Educación, del Departamento de Informática e Ingeniería de Sistemas de la Universidad de Zaragoza y por profesores del área de 
Tecnología y del área de Matemáticas de dos Institutos de Educación Secundaria de la Comunidad Autónoma de Aragón.

En la elaboración de dicho cuestionario se han controlado los posibles sesgos que afectarían a su validez y fiabilidad con el fin de mejorar el propio instrumento; la validación de contenido se garantizó presentando dicho cuestionario a 10 jueces expertos, quienes contaban con una pauta para evaluar de 1 a 5 el análisis de la congruencia y pertinencia de los ítems al propósito del cuestionario, así como también con un espacio para proponer cambios y/o sugerencias. Se eliminaron tres preguntas por no considerarlas coherentes en relación a los objetivos planteados. Y para determinar la fiabilidad o consistencia interna del cuestionario, se llevó a cabo una prueba piloto, en la cual participaron una pequeña muestra de 15 alumnos elegidos al azar de entre el grupo de la muestra participante, por lo que fueron alumnos de las tres Facultades y de los dos Grados de Magisterio en Educación Infantil y en Educación Primaria. Los resultados arrojaron un coeficiente de Alfa de Cronbach de 0,87, el cual excede el valor mínimo de 0,70 para determinar la confiabilidad de la prueba (DeVellis, 2003; Kline, 2000). Por esta razón, se puede establecer que existe una adecuada consistencia interna.

La versión final del cuestionario se incluye en el anexo I. Consta de 30 ítems, 20 de ellos tipo Likert de cuatro alternativas de respuesta y con posibilidad de añadir información adicional sobre las herramientas de Internet que usan los estudiantes. Y las diez preguntas restantes son de respuesta dicotómica.

Se trata de una prueba de administración colectiva, aunque también puede aplicarse de forma individual. En nuestro caso y como se explicará a continuación se ha pasado a 16 grupos de 60 estudiantes cada uno.

\section{Procedimiento}

Los profesores que hemos realizado esta investigación explicamos a la muestra en qué consistía el cuestionario, resolvimos sus dudas, y después de obtener su consentimiento, aplicamos dicho cuestionario a los 960 alumnos(as) en grupos de 60 estudiantes. En el apartado de datos personales se requirió solo la edad y el género, para mantener el anonimato de los discentes y evitar así respuestas contaminadas por la deseabilidad social.

Para evitar el gasto de papel, los estudiantes han contestado el cuestionario a través de la aplicación Google Docs mediante la creación de un formulario.

\section{Resultados}

La principal conclusión de este estudio, de carácter general pero muy reveladora, es que, aunque se piensa que los alumnos(as) que actualmente llegan a la Universidad son nativos digitales, sus competencias digitales en el uso de herramientas de Internet son escasas y mejorables.

Es cierto que todo el alumnado participante excepto un estudiante ha declarado tener ordenador personal, ya sea de sobremesa o portátil. Sin embargo, nuestra muestra 
conoce y usa mayoritariamente cuatro de las veinte herramientas de Internet sobre las que hemos preguntado en el cuestionario. Utilizan con gran frecuencia el navegador, el correo electrónico, la mensajería instantánea y las redes sociales generalistas, tal y como puede verse en la Tabla 2

Las herramientas que obtienen puntuaciones promedio más elevadas son: el correo electrónico ( $\mathrm{M}=2,87$ siendo la puntuación máxima 3, DT=0,27 muy baja dispersión), el navegador, con unas puntuaciones $(\mathrm{M}=2,84$, DT0=0,47), la herramienta de mensajería instantánea, con una $(\mathrm{M}=2,84, \mathrm{DT}=0,93)$ y las redes sociales generalistas, con una $(\mathrm{M}=2,36$ y una $\mathrm{DT}=0,97)$. Por el contrario el microbloging, las comunidades de contenido, las listas de distribución y los editores de HTML tienen puntuaciones medias de uso y conocimiento muy bajas, en torno a $\mathrm{M}=0,5$.

\section{Herramientas más usadas}

El correo electrónico, es la herramienta más usada y conocida por nuestros estudiantes, un 90,11\% la usa frecuentemente y la conoce. No hay prácticamente ningún alumno que no la conozca. Del navegador un 86,81\% de estudiantes hacen un uso frecuente y lo conocen. La mensajería instantánea ha obtenido resultados muy elevados en cuanto a su uso y conocimiento, así un $72,53 \%$ dicen que la usan frecuentemente. Creemos que este uso elevado de la mensajería instantánea es probablemente porque los estudiantes han confundido esta herramienta con el whatsApp, la herramienta de mensajería instantánea en los móviles, lo que cae fuera del objetivo de esta investigación. Las redes sociales generalistas es otra de las herramientas más usadas. Un 65,93\% de los estudiantes dicen que la usan frecuentemente y tan solo un $8,97 \%$ dice que no la conoce.

\begin{tabular}{lrrrr}
\hline & \multicolumn{1}{l}{$\begin{array}{l}\text { [Correo } \\
\text { electrónico] }\end{array}$} & $\begin{array}{r}\text { [Nensajería } \\
\text { [Navegador] }\end{array}$ & $\begin{array}{l}\text { [Redes sociales } \\
\text { instantánea] } \\
\text { generalistas] }\end{array}$ \\
\hline SUMA & 262 & 258 & 226 & 215 \\
\hline MEDIA & 2,87 & 2,84 & 2,48 & 2,36 \\
\hline DESVIACIÓN TíPICA & 0,27 & 0,47 & 0,94 & 0,98 \\
\hline Hago un uso frecuente & $90,11 \%$ & $86,81 \%$ & $72,53 \%$ & $65,93 \%$ \\
\hline Hago un uso esporádico & $8,79 \%$ & $10,99 \%$ & $9,89 \%$ & $13,19 \%$ \\
\hline Sé qué es pero no lo uso & $0,00 \%$ & $1,10 \%$ & $10,99 \%$ & $12,09 \%$ \\
\hline No sé qué es & $0,00 \%$ & $1,10 \%$ & $6,59 \%$ & $8,79 \%$ \\
\hline Porcentaje de uso & $98,90 \%$ & $97,80 \%$ & $82,42 \%$ & $79,12 \%$ \\
\hline Porcentaje de no uso & $0,00 \%$ & $2,20 \%$ & $17,58 \%$ & $20,88 \%$ \\
\hline
\end{tabular}

Tabla 2. Herramientas más usadas 


\section{Herramientas con un uso medio}

En este apartado hemos englobado todas aquellas herramientas que tienen un uso medio, entendiendo por tal aquellas cuyo porcentaje de uso frecuente está entre el $10 \mathrm{y}$ el 30 por ciento. Esas herramientas son las wikis, los programas de fuentes web, etiquetado social, las redes sociales profesionales y las llamadas por Internet (ver Tabla $3)$.

Las wikis tienen un uso frecuente por parte de nuestros estudiantes de un 27,47\%, un $37,36 \%$ hace un uso esporádico y más de un $15 \%$ no la conoce. ( $M=1,76, \mathrm{DT}=1,01)$. Creemos que esto es debido a que los estudiantes usan la Wikipedia, que es la wiki más popular.

Los programas de fuentes web tan solo los usan frecuentemente el $18,68 \%$ y un $30,77 \%$ hacen un uso esporádico, no lo usan más de un $40 \%$ y un $26,37 \%$ no saben qué son. La media de uso es de $\mathrm{M}=1,33$ sobre 3 y $\mathrm{DT}=1,08$.

El etiquetado social es muy desconocido para los estudiantes, pues un 57,14\% no saben lo que es, sólo un 13,19\% hace un uso frecuente del etiquetado. Su media de uso no llega a la unidad y su desviación típica es de 1,11.

El 58,24\% saben que son las redes sociales profesionales pero no las usan, sólo las usan de modo frecuente un 10,99\% de los estudiantes y de modo esporádico un 21,98\%. La media de uso debida fundamentalmente a este uso esporádico es de 1,3 sobre 3 y la desviación típica es de 0,77 lo que supone una baja dispersión.

La herramienta de llamadas telefónicas por internet obtiene, en cuanto al uso, unas puntuaciones similares a la anterior herramienta. Un 10,99\% hacen un uso frecuente de este tipo de llamadas, y un 35,16\% hace un uso esporádico. Un 48,35\%, es decir, casi la mitad de los estudiantes encuestados saben qué es esta herramienta pero no la usan.

\begin{tabular}{|c|c|c|c|c|c|}
\hline & [Wiki] & $\begin{array}{l}\text { [Fuente } \\
\text { web] }\end{array}$ & $\begin{array}{l}\text { [Etiquetado } \\
\text { social] }\end{array}$ & $\begin{array}{l}\text { [Redes sociales } \\
\text { profesionales] }\end{array}$ & $\begin{array}{l}\text { [Llamadas } \\
\text { telefónicas por } \\
\text { Internet] }\end{array}$ \\
\hline SUMA & 161 & 127 & 28 & 93 & 138 \\
\hline MEDIA & 1,77 & 1,33 & 0,83 & 1,37 & 1,53 \\
\hline DESVIACIÓN TÍPICA & 1,01 & 1,08 & 1,12 & 0,78 & 0,74 \\
\hline Hago un uso frecuente & $27,47 \%$ & $18,68 \%$ & $13,19 \%$ & $10,99 \%$ & $10,99 \%$ \\
\hline Hago un uso esporádico & $37,36 \%$ & $30,77 \%$ & $14,29 \%$ & $21,98 \%$ & $35,16 \%$ \\
\hline Sé qué es pero no lo uso & $19,78 \%$ & $21,98 \%$ & $14,29 \%$ & $58,24 \%$ & $48,35 \%$ \\
\hline No sé qué es & $15,38 \%$ & $26,37 \%$ & $57,14 \%$ & $7,69 \%$ & $4,40 \%$ \\
\hline Porcentaje de uso & $64,83 \%$ & $49,45 \%$ & $27,48 \%$ & $32,97 \%$ & $46,15 \%$ \\
\hline Porcentaje de no uso & $35,16 \%$ & $48,35 \%$ & $71,43 \%$ & $65,93 \%$ & $52,75 \%$ \\
\hline
\end{tabular}

Tabla 3. Herramientas con uso medio 


\section{Herramientas con un uso bajo}

En este apartado hemos englobado aquellas herramientas que tienen un uso bajo, entendiendo por tal aquellas cuyo porcentaje de uso frecuente es inferior al 10 por ciento. Esas herramientas son los compresores de archivos, las de ofimática en la nube, foros, grupos de noticias, blogs, microbloging, comunidades de contenido, listas de distribución y los editores de HTML (ver Tabla 4, parte 1, parte 2 y parte 3).

Los compresores de archivos son usados frecuentemente tan solo por un 8,79\% de los estudiantes y conocida aunque usada con poca frecuencia en un 32,97\%, y conocida pero no usada en otro $32,97 \%$, no la conocen un $25,27 \%$ y su media de uso es de un 1,25 sobre 3 con una desviación típica de un 0,93.

La ofimática en la nube se recoge en el cuestionario en tres herramientas que son los editores de textos, hojas de cálculo y los programas de presentaciones. Los resultados de uso y conocimiento de los editores y de los programas de presentaciones son muy similares, dando una media de uso de 0,91 sobre tres y una desviación típica muy parecida en ambas herramientas: Un 0,89 en la herramienta de editores de texto en la nube y un 0,86 en la herramienta de presentaciones en la nube. Las hojas de cálculo en la nube también tienen un uso muy poco frecuente, tan solo un 3,3\% dice usarlas frecuentemente, un 9,89\% dice usarlas pero con poca frecuencia. El 52,75\% las conoce pero no las usa y un $34,07 \%$ ni tan siquiera las conoce. ( $M=0,82$ sobre 3 y $D T=0,73$ ).

Los grupos de noticias tienen un porcentaje de uso frecuente de un 5,49\% y un porcentaje de uso poco frecuente de un $28,57 \%$. El 58,24\% lo conocen pero no lo usan, y un $6,59 \%$ no lo conocen. La media de uso es de un 1,33 sobre tres que es el valor máximo y la desviación típica es de 0,67.

Los foros tienen un $4,4 \%$ de porcentaje de uso frecuente por parte de los estudiantes, y un $26,37 \%$ de uso poco frecuente. El 69,23\% de los jóvenes no los usan.

Los blogs tienen un uso muy bajo por parte de los estudiantes, tan solo un 2,20\% dicen usarlos frecuentemente, un $28,57 \%$ los usan con poca frecuencia, un $65,93 \%$ los conocen pero no los usan y un $2,20 \%$ no los conocen. La media de uso es de un 1,3 sobre 3 y la desviación de 0,55.

Las herramientas de microbloging, comunidades de contenido y listas de distribución tienen un porcentaje de alumnos que las usan frecuentemente de tan solo un $1,10 \%$ y la mayoría no las conocen.

El editor de HTML nadie lo usa frecuentemente, un 10,99\% hace un uso esporádico del mismo, y un 32,97\% lo conoce pero no lo usa. Un 54,95\% no lo conoce. 


\begin{tabular}{|c|c|c|c|c|}
\hline & $\begin{array}{l}\text { [Compresor } \\
\text { de archivos] }\end{array}$ & $\begin{array}{l}\text { [Editor de texto } \\
\text { en la nube] }\end{array}$ & $\begin{array}{l}\text { [Hoja de } \\
\text { cálculo en la } \\
\text { nube] }\end{array}$ & $\begin{array}{l}\text { [Presentación } \\
\text { en la nube] }\end{array}$ \\
\hline SUMA & 114 & 82 & 75 & 7 \\
\hline MEDIA & 1,25 & 0,91 & 0,82 & 0,91 \\
\hline DESVIACIÓN TÍPICA & 0,93 & 0,89 & 0,74 & 0,86 \\
\hline Hago un uso frecuente & $8,79 \%$ & $8,79 \%$ & $3,30 \%$ & $6,59 \%$ \\
\hline Hago un uso esporádico & $32,97 \%$ & $8,79 \%$ & $9,89 \%$ & $12,09 \%$ \\
\hline Sé qué es pero no lo uso & $32,97 \%$ & $47,25 \%$ & $52,75 \%$ & $45,05 \%$ \\
\hline No sé qué es & $25,27 \%$ & $35,16 \%$ & $34,07 \%$ & $34,07 \%$ \\
\hline Porcentaje de uso & $41,76 \%$ & $17,58 \%$ & $13,19 \%$ & $18,68 \%$ \\
\hline Porcentaje de no uso & $58,24 \%$ & $82,41 \%$ & $86,82 \%$ & $79,12 \%$ \\
\hline
\end{tabular}

Tabla 4 (parte 1). Herramientas de uso bajo

\begin{tabular}{lrrrr}
\hline & $\begin{array}{l}\text { [Grupos de } \\
\text { noticias] }\end{array}$ & [Foros] & [Blog] & [Microblogging] \\
\hline SUMA & 120 & 123 & 117 & 32 \\
\hline MEDIA & 1,33 & 1,19 & 1,31 & 0,35 \\
\hline DESVIACIÓN TíPICA & 0,68 & 0,57 & 0,56 & 0,66 \\
\hline Hago un uso frecuente & $5,49 \%$ & $4,40 \%$ & $2,20 \%$ & $1,10 \%$ \\
\hline Hago un uso esporádico & $28,57 \%$ & $26,37 \%$ & $28,57 \%$ & $6,59 \%$ \\
\hline Sé qué es pero no lo uso & $58,24 \%$ & $69,23 \%$ & $65,93 \%$ & $18,68 \%$ \\
\hline No sé qué es & $6,59 \%$ & $0,00 \%$ & $2,20 \%$ & $73,63 \%$ \\
\hline Porcentaje de uso & $34,06 \%$ & $30,77 \%$ & $30,77 \%$ & $7,69 \%$ \\
\hline Porcentaje de no uso & $64,83 \%$ & $69,23 \%$ & $68,13 \%$ & $92,31 \%$ \\
\hline
\end{tabular}

Tabla 4 (parte 2). Herramientas de uso bajo

\begin{tabular}{lrrr}
\hline & $\begin{array}{c}\text { [Comunidades de } \\
\text { contenidos] }\end{array}$ & $\begin{array}{r}\text { [Listas de } \\
\text { distribución] }\end{array}$ & $\begin{array}{r}\text { [Editor de } \\
\text { HTML] }\end{array}$ \\
\hline SUMA & 47 & 51 & 50 \\
\hline MEDIA & 0,52 & 0,56 & 0,56 \\
\hline DESVIACIÓN TÍPICA & 0,78 & 0,62 & 0,56 \\
\hline Hago un uso frecuente & $1,10 \%$ & $1,10 \%$ & $0,00 \%$ \\
\hline Hago un uso esporádico & $14,29 \%$ & $3,30 \%$ & $10,99 \%$ \\
\hline Sé qué es pero no lo uso & $19,78 \%$ & $46,15 \%$ & $32,97 \%$ \\
\hline No sé qué es & $64,84 \%$ & $49,45 \%$ & $54,95 \%$ \\
\hline Porcentaje de uso & $15,39 \%$ & $4,40 \%$ & $10,99 \%$ \\
\hline Porcentaje de no uso & $84,62 \%$ & $95,60 \%$ & $87,92 \%$ \\
\hline
\end{tabular}

Tabla 4 (parte 3). Herramientas de uso bajo 


\section{Conclusiones}

Si definimos la competencia digital como el uso crítico y frecuente de las herramientas que hemos incluido en este estudio, la principal conclusión que podemos extraer es que, contrariamente a lo que se supone, los alumnos de Magisterio no tienen una elevada competencia digital, a pesar de ser considerados nativos digitales. En este sentido, Gutiérrez, Palacios y Torrego (2010) incidían que no todos los alumnos de Magisterio son usuarios habituales de TIC, incluso llegaron a afirmar que los «nativos» digitales de los que tanto se ha hablado parece que no han llegado todavía a la universidad española.

Frente a las 20 herramientas revisadas en este trabajo, hemos constatado que los alumnos(as) solo hacen un uso frecuente de cuatro de ellas: El navegador, el correo electrónico, la mensajería instantánea y las redes sociales generalistas. En cuanto el uso y dominio del navegador también fue constatado en el estudio realizado por Roig y Pascual (2012). La explicación de este uso es sencilla. El navegador en el ordenador es de uso obligatorio como puerta de entrada al resto de las herramientas, y el correo electrónico es el instrumento de comunicación básico en muchos entornos, entre ellos, el entorno académico. Por eso, esas dos herramientas ocupan los dos primeros lugares de uso. En cuanto a las otras dos herramientas, su uso se sitúa más en el entorno del ocio que del trabajo diario. Las redes sociales generalistas sirven para mantenerse en contacto con amigos y compañeros. En cuanto a la mensajería instantánea, creemos que los alumnos(as) no han pensado en su uso en el ordenador sino en el uso que hacen a través de sus teléfonos móviles, a través de aplicaciones como WhatsApp, Hangouts, etc.

En nuestra opinión, estos resultados no son sorprendentes ya que la mayor parte de las herramientas que estamos considerando son relativamente recientes (por ejemplo, la herramienta de ofimática en la nube Google Docs está disponible en producción desde el año 2009) y la implantación de las mismas no se consigue de forma abrupta sino que llega a través de un proceso gradual que puede durar años.

Siendo firmes defensores del uso de las nuevas tecnologías en el entorno laboral, los resultados muestran que debe hacerse un esfuerzo de formación, orientado a que los alumnos(as) desarrollen la competencia digital, ya que una parte de ella implica el uso de las distintas herramientas, tal y como se recoge en la OCDE (2002). Así mismo, incidimos en la importancia de que desarrollen las distintas herramientas que se han recogido en este estudio ya que pueden facilitarles su futuro trabajo en las escuelas. Pues dicho futuro pasa por una redefinición de la labor docente y de su profesionalización donde la competencia digital juega un papel fundamental para responder a los desafíos de innovación dentro de las aulas desde la calidad y mejora de la educación.

Por ello, insistimos en la importancia de introducir como parte de la formación inicial del docente estas herramientas, y prepararles para que sean capaces de integrarlas pedagógicamente. Además la destreza en el uso de herramientas de Internet va a reverberar sobre el desarrollo de otra competencia clave, que es aprender $a$ aprender, necesaria para el aprendizaje a lo largo de la vida, la formación continua del 
maestro(a) y su reciclaje. Coincidimos con Gutiérrez, Palacios y Torrego (2010) en que la formación inicial es el momento ideal para predisponer positivamente a los maestros(as) hacia la integración curricular de las TIC, ya que la formación del profesorado en ejercicio suele estar más encaminada a modificar actuaciones y prácticas concretas en un momento dado que a generar actitudes abiertas y favorables tanto a la innovación educativa con TIC, como a la cada día más imprescindible alfabetización digital.

Las propuestas formativas que lanzamos para orientar la formación inicial de profesores en el uso de las TIC, tomando como referente la sociedad del conocimiento y la globalización en la que nos encontramos inmersos, se concretan en:

- Introducir como contenidos de trabajo en las guías docentes de las asignaturas de “Materiales y Recursos” en el Grado de Magisterio en Educación Infantil y la asignatura de "la Educación en la Sociedad del Conocimiento" en el Grado de Magisterio en Educación Primaria estos contenidos: compresores de archivos, herramientas de ofimática en la nube, foros, grupos de noticias, blogs, microbloging, comunidades de contenido, listas de distribución y los editores de HTML. Por ser las herramientas que los alumnos(as) menos usan y desconocen y, por lo tanto, en las que están menos capacitados.

- Intensificar la dedicación en las asignaturas anteriormente señaladas de los contenidos de: las wikis, los programas de fuentes web, el etiquetado social, las redes sociales profesionales y las llamadas por Internet, entre otros.

En definitiva, lo que se pretende es dotar al docente de referentes y orientaciones en el uso de estas herramientas que le permitan diseñar ambientes propicios para el aprendizaje e innovar en el aula para contribuir a la calidad de la educación.

\section{Agradecimientos}

Queremos agradecer a Ángel de Miguel y Áurea Rodríguez su trabajo en la elaboración del test que hemos pasado a los alumnos.

\section{Referencias bibliográficas}

ALA-MUTKA, K., PUNIE, Y. Y REDECKER, C. (2008). Digital competence for lifelong learning. Luxembourg: Office for Official Publications of the European Communities

AREA, M. (2005). La escuela y la sociedad de la información. En VV.AA.: Nuevas tecnologías, globalización y migraciones. (pp. 39-54). Barcelona: Editorial Octaedro. 
BARRANTES, G., CASAS, L. Y LUENGO, R. (2011). Obstáculos percibidos para la integración de las TIC por los profesores de Infantil y Primaria en Extremadura. Pixel-Bit. 39, 83-94.

BAUTISTA, A. (2000). Tres temas tecnológicos para la formación del profesorado. Revista de Educación, 322, 30-42.

CABERO, J. (2004). Formación del profesorado en TIC. El gran caballo de batalla. En Comunicación y Pedagogía. Tecnologías y recursos didácticos, 195 (pp. 27-31). Recuperado de http://www.atei.es/uao/panorama/15.pdf

COLL, C., MAURI, T. Y ONRUBIA, J. (2008). Análisis de los usos reales de las TIC en contextos educativos formales: una aproximación sociocultural. Revista Electrónica de Investigación Educativa, $10 \quad$ (1), 1 18.http://redie.uabc.mx/contenido/vol10no1/contenido-coll2.pdf

DEVELLIS, R. F. (2003). Scale development: Theory and applications (2a ed.). Newbury Park, CA: Sage.

GRANADOS, J.M. (2007). Los programas multimedia en los procesos de integración curricular de las tecnologías digitales. Revista Interuniversitaria de Formación del Profesorado, 21 (1), 127-143.

GUTIÉRREZ, A. (2008). Las Tic en la formación del maestro. "Realfabetización" digital del profesorado. Revista Interuniversitaria de Formación del Profesorado, $63(22,3), 191-206$.

GUTIÉRREZ, A. (2003). Multimedia Authoring as a fundamental principle of literacy and teacher training in the Information Age. En B. Duncan y K. Tyner (Eds.): Visions/Revisions. Moving Forward with Media Education. (pp. 391-422). Madison: National Telemedia Council.

GUTIÉRREZ, A; PALACIOS, A. Y TORREGO, L. (2010): La formación de los futuros maestros y la integración de las TIC en la educación: anatomía de un desencuentro. Revista de Educación, 352, 267-293

INSTITUTO DE TECNOLOGÍAS EDUCATIVAS (ITE). (2011). Informe Competencia digital. Ministerio de Educación, cultura y deporte. Recuperado de http://recursostic.educacion.es/blogs/europa/media/blogs/europa/informes/Compete ncia_Digital_Europa_ITE_marzo_2011. pdf.

KLINE, P. (2000). The handbook of psychological testing ( $\left.2^{a} e d.\right)$. New York: Routledge.

LEY ORGÁNICA 2/2006, DE 3 DE MAYO, de Educación. (B.O.E. 4-5- 2006).

LEY ORGÁNICA 8/2013, de 9 de diciembre, para la mejora de la calidad educativa. (B.O.E. 10-12-2013)

MARÍN DÍAZ, V. (2012). La competencia digital del alumno del siglo XXI. Actas del Congreso Internacional EDUTEC. (pp.192-210). 
MARQUÈS, P. (2003). Las competencias didáctico-digitales de los formadores en la Era Internet. Recuperado de http://dewey.uab.es/pmarques/simposium.htm.

MUÑOZ, A. (Coord.) (2010). Competencias básicas en Educación Infantil. Clave XXI, 2. Recuperado de http://www.clave21.es/files/articulos/CompetenciasEI_0.pdf.

ORDEN DE 28 DE MARZO DE 2008, por la que se aprueba el currículo de la Educación Infantil.

ORDEN DE 9 DE MAYO de 2007, por la que se aprueba el currículum de la Educación Primaria.

ORDEN DE 16 DE JUNIO de 2014, de la Consejera de Educación, Universidad, Cultura y Deporte, por la que se aprueba el currículo de la Educación Primaria y se autoriza su aplicación en los centros docentes de la Comunidad Autónoma de Aragón.

OCDE (2002). Définitions et sélection des compétences. Fondements théoriques et conceptuels. Documents de strategie. DEELSA/ED/CERI/CD

PRENSKY, M. (2001). Digital Natives, Digital Immigrants. En On the Horizon, NCB University Press, Vol. 9 No. 5, October 2001.

REAL DECRETO 1513/2006, de 7 de Diciembre, por el que se establecen las enseñanzas mínimas de la Educación Primaria.

REAL DECRETO 1630/2006, de 29 de diciembre, por el que se establece el currículo y las enseñanzas mínimas del segundo ciclo de la Educación Infantil

ROIG VILA, R. Y PASCUAL LUNA, A. M. (2012). Las competencias digitales de los futuros docentes. Un análisis con estudiantes de Magisterio de Educación Infantil de la Universidad de Alicante. @tic. revista d'innovació educativa, 9, 53-60. Fecha de consulta: 5 de enero de 2015 


\section{Anexo I}

\section{Test de uso de herramientas de Internet}

\section{Edad:}

sexo:

En las siguientes preguntas contesta con un SI o un NO

Tienes ordenador en casa:

Dispones de conexión a Internet en tu hogar:

Tienes ordenador portátil para uso exclusivo:

Utilizas ordenador propio para realizar los trabajos de la carrera:

Utilizas los ordenadores de la universidad o de otros lugares por no tener ordenador propio:

Tienes teléfono móvil:

Tienes conexión a Internet en el móvil:

Tienes cuenta de correo:

En cada una de las filas, pon una única marca en la columna que proceda de acuerdo con tu uso de la herramienta 


\begin{tabular}{|c|c|c|c|c|c|c|}
\hline Herramienta & $\begin{array}{l}\text { No sé } \\
\text { qué es }\end{array}$ & $\begin{array}{l}\text { Sé qué es } \\
\text { pero } \\
\text { no lo uso }\end{array}$ & $\begin{array}{l}\text { Hago un uso } \\
\text { esporádico }\end{array}$ & $\begin{array}{l}\text { Hago un uso } \\
\text { frecuente }\end{array}$ & \multicolumn{2}{|c|}{$\begin{array}{l}\text { Información adicional sobre la } \\
\text { herramienta (PARA LAS } \\
\text { HERRAMIENTAS QUE USAS) }\end{array}$} \\
\hline Navegador & & & & & Nombre del navegador & \\
\hline $\begin{array}{l}\text { Compresor de } \\
\text { archivos }\end{array}$ & & & & & $\begin{array}{l}\text { Nombre del compresor } \\
\text { de archivos }\end{array}$ & \\
\hline $\begin{array}{l}\text { Correo } \\
\text { electrónico }\end{array}$ & & & & & $\begin{array}{l}\text { Nombre del proveedor } \\
\text { de correo }\end{array}$ & \\
\hline $\begin{array}{l}\text { Mensajería } \\
\text { instantánea }\end{array}$ & & & & & $\begin{array}{l}\text { Nombre de la aplicación } \\
\text { de mensajería }\end{array}$ & \\
\hline Foros & & & & & & \\
\hline $\begin{array}{l}\text { Grupos de } \\
\text { noticias }\end{array}$ & & & & & & \\
\hline $\begin{array}{l}\text { Listas de } \\
\text { distribución }\end{array}$ & & & & & & \\
\hline $\begin{array}{l}\text { Editores de } \\
\text { HTML }\end{array}$ & & & & & $\begin{array}{l}\text { Nombre del editor de } \\
\text { HTML }\end{array}$ & \\
\hline Blogs & & & & & $\begin{array}{l}\text { Mantengo un blog } \\
\text { Nombre del sitio de } \\
\text { blogs }\end{array}$ & $\begin{array}{l}\text { Leo [ ] } \\
\text { Escribo [ ] }\end{array}$ \\
\hline Fuentes web & & & & & $\begin{array}{l}\text { Nombre del programa de } \\
\text { fuentes web }\end{array}$ & \\
\hline Wikis & & & & & Nombre de la wiki & \\
\hline $\begin{array}{l}\text { Editor de texto } \\
\text { en la nube }\end{array}$ & & & & & $\begin{array}{l}\text { Nombre del editor de } \\
\text { texto en la nube }\end{array}$ & \\
\hline $\begin{array}{l}\text { Hoja de cálculo } \\
\text { en la nube }\end{array}$ & & & & & $\begin{array}{l}\text { Nombre de la hoja de } \\
\text { cálculo en la nube }\end{array}$ & \\
\hline $\begin{array}{l}\text { Presentación en } \\
\text { la nube }\end{array}$ & & & & & $\begin{array}{l}\text { Nombre del programa de } \\
\text { presentación en la nube }\end{array}$ & \\
\hline $\begin{array}{l}\text { Llamadas } \\
\text { telefónicas } \\
\text { por Internet }\end{array}$ & & & & & $\begin{array}{l}\text { Nombre del programa de } \\
\text { llamadas telefónicas }\end{array}$ & \\
\hline $\begin{array}{l}\text { Redes sociales } \\
\text { generalistas }\end{array}$ & & & & & $\begin{array}{l}\text { Participo en una red } \\
\text { social generalista } \\
\text { Nombre de la red social } \\
\end{array}$ & $\begin{array}{l}\text { Leo [ ] } \\
\text { Escribo [ ] }\end{array}$ \\
\hline $\begin{array}{l}\text { Redes sociales } \\
\text { profesionales }\end{array}$ & & & & & $\begin{array}{l}\text { Participo en una red } \\
\text { social profesional } \\
\text { Nombre de la red } \\
\text { profesional }\end{array}$ & $\begin{array}{l}\text { Leo [ ] } \\
\text { Escribo [ ] }\end{array}$ \\
\hline Microblogging & & & & & $\begin{array}{l}\text { Uso el microblogging } \\
\text { Nombre del sitio de } \\
\text { microblogging }\end{array}$ & $\begin{array}{l}\text { Leo [ ] } \\
\text { Escribo [ ] }\end{array}$ \\
\hline $\begin{array}{l}\text { Comunidades de } \\
\text { contenidos }\end{array}$ & & & & & $\begin{array}{l}\text { Nombre de las } \\
\text { comunidades de } \\
\text { contenidos }\end{array}$ & \\
\hline $\begin{array}{l}\text { Etiquetado } \\
\text { social }\end{array}$ & & & & & $\begin{array}{l}\text { Nombre de las } \\
\text { herramientas de } \\
\text { etiquetado social }\end{array}$ & \\
\hline
\end{tabular}




\section{Correspondencia con los autores}

Marta LIESA ORÚS

Facultad de Ciencias Humanas y de la Educación.

C/ Valentín Carderera, 4,

22003 Huesca

e-mail: martali@unizar.es

Sandra VÁZQUEZ TOLEDO

Dirección laboral: Facultad de Ciencias Humanas y de la Educación.

C/ Valentín Carderera, 4,

22003 Huesca

e-mail: svaztol@unizar.es

Jorge LLORET GAZO

Facultad de Ciencias

Campus San Francisco

50009 Zaragoza

e-mail: jlloret@unizar.es 